\title{
Análise epidemiológica das oclusões arteriais agudas dos membros inferiores em hospital quaternário
}

\author{
Epidemiological analysis of critical lower limb ischemia in a quaternary hospital
}

\author{
Melissa Andreia de Moraes-Silva ${ }^{1}$, Gabriela Bueno ${ }^{2}$, Seleno Glauber de Jesus-Silva ${ }^{3}$, \\ Arturo Eduardo Krupa ${ }^{4}$, Rodolfo Souza Cardoso 5
}

\begin{abstract}
Moraes-Silva MA, Bueno G, Jesus-Silva SG, Krupa AE,Cardoso RS. Análise epidemiológica das oclusões arteriais agudas dos membros inferiores em hospital terciário / Epidemiological analysis of critical lower limb ischemia in a terciary hospital. Rev Med (São Paulo). 2017 out.-dez.;96(4):264-70.

RESUMO: Introdução: a oclusão arterial aguda (OAA) dos membros inferiores é qualquer diminuição ou piora súbita na perfusão do membro, que determine ameaça à sua viabilidade. Objetivos: analisar as características epidemiológicas dos pacientes portadores de OAA em um hospital terciário. Casuística e Métodos: estudo retrospectivo e transversal, com revisão de prontuários de pacientes internados devido OAA em membros inferiores ocorridas de 2013 a 2016. Foram analisadas as

cirúrgico mais comum foi a tromboembolectomia, realizada em $39,7 \%$, seguido da revascularização endovascular, em 12,70\%. Conclusão: o tratamento endovascular ainda é pouco indicado nos casos de OAA, recaindo na cirurgia convencional a opção de revascularização imediata do membro. Os pacientes portadores de OAA de membro inferior possuem alta prevalência de fatores de risco para doença aterosclerótica, apesar de os mesmos não estarem diretamente relacionados à perda do membro.
\end{abstract} variáveis: idade, sexo, comorbidades (diabete melito, hipertensão arterial sistêmica, cardiopatias), tabagismo, tratamento e evolução durante a internação. Foi utilizado o teste do Quiquadrado para comparação entre os grupos. Resultados: foram avaliados 51 pacientes $(52,9 \%$ sexo masculino), com média de idade de 68 anos em ambos os sexos. Entre as comorbidades, $78,4 \%$ eram hipertensos, $66,7 \%$ tabagistas, $35,3 \%$ portadores de fibrilação atrial, e 27,4\% diabéticos. Dezesseis casos evoluíram para amputação, sendo 14 amputações maiores. Não houve diferença estatística significativa nas comorbidades entre os grupos que evoluíram ou não para amputação. No entanto, os pacientes classificados como Rutherford III apresentaram índices maiores de amputação $(\mathrm{p}=0.0043)$. $\mathrm{O}$ procedimento
Descritores: Embolia e trombose; Isquemia; Doença arterial periférica/epidemiologia; Extremidade inferior; Lower extremity.

ABSTRACT: Introduction: acute limb ischemia (ALI) is any sudden decrease or worsening in limb perfusion that threatens its viability. Objective: to analyze the epidemiological characteristics of patients with ALI in a terciary hospital. Methods: retrospective and cross-sectional study, with review of medical records of hospitalizations due to ALI in lower limbs occurring from 2013 to 2016. The following variables were analyzed: age, sex, comorbidities (diabetes melitus, systemic arterial hypertension,

Trabalho apresentado no III Congresso Brasileiro das Ligas de Cirurgia, São Paulo, SP, 27 jan. 2017.

1.Faculdade de Medicina de Itajubá, Itajubá, MG, BR. Cirurgiã Vascular. Professora Adjunta da Disciplina de Cirurgia Vascular. Mestre em Ciências pela Universidade Federal de São Paulo - UNIFESP. E-mail: meldemoraes@gmail.com

2.Faculdade de Medicina de Itajubá, Itajubá, MG, BR. Acadêmica do 6º ano do Curso de Medicina da FMIt. E-mail: gabi.bueno93@ gmail.com.

3.Faculdade de Medicina de Itajubá, Itajubá, MG, BR. Cirurgião Vascular. Professor Assistente da Disciplina de Clínica Cirúrgica. E-mail: selenoglauber@gmail.com.

4.Faculdade de Medicina de Itajubá, Itajubá, MG, BR. Cirurgião Vascular. Professor Titular da Disciplina de Cirurgia Vascular. E-mail: arturoekrupa@gmail.com.

5.Faculdade de Medicina de Itajubá, Itajubá, MG, BR. Cirurgião Vascular. Professor Titular da Disciplina de Clínica Cirúrgica. E-mail: rodolfose@globo.com.

Endereço para correspondência: Melissa Andreia de Moraes-Silva. Avenida Renó Júnior, 368. Itajubá, MG, BR. CEP: $37502-138$.

Email: meldemoraes@gmail.com. 
chronic heart diseases), smoking, treatment and in-hospital outcomes. Chi-square test was used to compare groups. Results: fifty-one patients $(52.9 \%$ male) were studied, with average age of $68 \mathrm{y}$ in both sexes. Among comorbidities, $78.4 \%$ were hypertensive, $66.7 \%$ were smokers, $35.3 \%$ had atrial fibrillation, and $27.4 \%$ were diabetics. Sixteen cases evolved to amputation, of which 14 were major amputations. There was no statistically significant difference on the comorbidities between the groups that evolved or not to amputation. However, patients classified as Rutherford III had higher rates of amputation $(\mathrm{p}=0.0043)$. The

\section{INTRODUÇÃO}

A doenças cardiovasculares são as principais causas de morte em ambos os sexos no Brasil, sendo responsáveis por cerca de $20 \%$ de todas as mortes acima de 30 anos. As estatísticas do Ministério da Saúde apontam que ocorreram 962.931 mortes em pessoas com idade maior de 30 anos em 2009. As causas cardiovasculares atribuíveis à aterosclerose, isoladamente, foram responsáveis por 193.309 mortes $^{1}$.

A oclusão arterial aguda (OAA) dos membros inferiores é uma condição clínica que cresce em frequência com o envelhecimento da população e com o aumento na prevalência das doenças cardiovasculares associadas à aterosclerose. Estima-se que sua incidência hospitalar seja de 32,3 casos/100.000 indivíduos/ano ${ }^{2}$.

Pode ser de etiologia embólica, em sua grande maioria cardiogênica, ou trombótica, nos portadores de doença arterial obstrutiva periférica (DAOP). O quadro clínico é amplo, descrito como dor súbita intensa, parestesia e paralisia, frialdade e palidez da extremidade acometida, além da diminuição ou até mesmo a ausência de pulsos periféricos nas regiões distais à oclusão ${ }^{3,4}$.

A DAOP, por sua vez, caracteriza-se por um déficit gradual de fluxo sanguíneo, geralmente devido a processo oclusivo aterosclerótico dos membros inferiores. A claudicação intermitente, ou seja, dor ou câimbra em grupos musculares após atividade física, é a manifestação clínica mais comum da $\mathrm{DAOP}^{5,6}$. A progressão da DAOP de claudicação para dor em repouso ou úlcera isquêmica pode ocorrer de forma gradual, mas é resultante de um ou mais episódios que pioram a isquemia existente.

Entretanto, as OAA dos membros inferiores podem ocorrer como primeiro evento, em um paciente previamente assintomático. A piora aguda da claudicação intermitente (por exemplo, de 500 metros para 50 metros) pode ocorrer como resultado de um evento agudo, todavia não é comumente aceita como OAA. Portanto, é definido que a OAA dos membros inferiores é qualquer diminuição ou piora súbita na perfusão do membro, que determine potencial ameaça à sua viabilidade?

Os fatores de risco mais significativos para o desenvolvimento de doença arterial periférica sintomática são o tabagismo e diabete melito, seguidos pela hipertensão arterial e pela dislipidemia. A ausência de fatores de risco most common surgical procedure was thromboembolectomy, performed in $39.7 \%$, followed by endovascular revascularization, in $12.7 \%$. Conclusion: endovascular treatment is still poorly indicated in ALI, while conventional surgery is still the firstline therapy. Patients with ALI have higher prevalence of atherosclerosis risk factors, although they are not directly related to limb loss.

Keywords: Embolism and thrombosis; Ischemia; peripheral arterial disease/epidemiology.

é protetora, bem como determinados hábitos, como a prática de exercícios físicos. Os fatores genéticos parecem ter uma influência na doença arterial periférica, mas os dados são limitados. Em um estudo conduzido por Wassel et al. ${ }^{8}$, a história familiar de doença arterial periférica foi independentemente e fortemente associada com a prevalência e gravidade das manifestações clínicas nos pacientes. Isso indica um papel do componente genético, ou outros fatores ambientais compartilhados - ou ambos - na contribuição para a gênese dessa patologia. Também foi demonstrado que a hipercolesterolemia familiar, uma desordem genética, é relacionada com alto risco de doenças periféricas arteriais. No entanto, a influência da história familiar como fator de risco ainda se constitui em uma área ativa de investigação ${ }^{7}$.

O diagnóstico diferencial da OAA é de extrema importância e pode ser dividido em três grupos distintos: a) condições que se assemelham às da OAA, aonde se incluem, por exemplo, a trombose venosa profunda e as neuropatias compressivas agudas; b) causas não arterioscleróticas de OAA, incluindo traumatismo arterial, embolia, dissecção aórtica ou arterial, vasoespasmo com trombose, entre outras; c) causas de oclusão arterial aguda em pacientes com arteriosclerose, dentre os quais podem ser mencionados a trombose de artéria com placa aterosclerótica, aneurismas trombosados, trombose de ponte arterial e ateroembolismo ${ }^{7}$.

O presente estudo teve por objetivo analisar a epidemiologia das OAA dos membros inferiores em um hospital de ensino quaternário, correlacionando as principais variáveis clínicas, terapêutica empregada e resultados intra-hospitalares.

\section{CASUÍSTICA E MÉTODOS}

Estudo retrospectivo e transversal, com revisão de prontuários médicos de internações por OAA em membros inferiores ocorridas no Hospital Escola de Itajubá-MG, no período de janeiro de 2013 a fevereiro de 2016. O trabalho foi aprovado pelo Comitê de Ética em Pesquisa da instituição sob o número 1.550.931.

O dimensionamento para o cálculo da amostra mínima necessária foi realizado utilizando grau de confiança de $95 \%$, erro máximo absoluto de $5 \%$ e prevalência de fatores de risco em pacientes entre 30 e $68 \%$. 
Obteve-se valor de 53 indivíduos como amostra mínima significativa. Dois prontuários não foram encontrados no arquivo do hospital, sendo a amostra final de 51 casos.

A distinção dos casos estudados foi realizada somente através das características clínicas, uma vez que nem todos os pacientes foram submetidos a exames de imagem, sendo categorizadas segundo a Classificação de Rutherford ${ }^{9}$ como viável (grau I, ou seja, membro sem ameaça imediata), viabilidade ameaçada (grau IIa, correspondente à membro marginalmente ameaçado ou grau IIb, equivalente a ameaça imediata), e lesão tecidual irreversível (grau III, representando membro inviável).

Foram analisados idade, sexo, presença de hipertensão arterial sistêmica (PA>140x90mmHg), diabete melito (uso de hipoglicemiantes ou glicemia de jejum $>126 \mathrm{mg} / \mathrm{dL}$ ), antecedentes de cardiopatias, dislipidemia (uso de estatina ou LDL $>160 \mathrm{mg} / \mathrm{dL}$ ), doença coronariana ou infarto agudo do miocárdio prévio, insuficiência renal crônica (creatinina $>1,6 \mathrm{mg} / \mathrm{dL}$ ou clearance de creatinina $<$ $60 \mathrm{ml} / \mathrm{min}$ ) e insuficiência cardíaca congestiva (definida pela história clínica). Além disso, foram registrados os hábitos de vida, englobando tabagismo e etilismo, os resultados de arteriografias, bem como o tratamento instituído, o tipo de cirurgia empregada e a evolução apresentada durante a internação $0^{7,10,11}$. Dados sobre a prática de exercícios físicos e os antecedentes familiares não estavam disponíveis em todos os prontuários, portanto não foram incluídos no estudo.

Todos os sujeitos estudados apresentaram como diagnóstico de internação a OAA de membros inferiores. Não foram incluídos os casos em que a etiologia da oclusão estava relacionada a outros diagnósticos ou em que os exames que não demonstravam informações conclusivas ${ }^{13}$.

Os dados dos pacientes foram computados em planilha eletrônica, com as respectivas informações demográficas, quadro clínico e fatores de risco. A análise entre variáveis contínuas foi realizada por meio do teste $\mathrm{T}$ de Student, e entre as variáveis categóricas, pelo Qui-quadrado, considerando $\mathrm{p}<0,05$ como valor significativo $^{14,15}$.

\section{RESULTADOS}

Dos 51 casos estudados, 24 eram do sexo feminino. A média de idade de todos os pacientes foi de 68 anos (mín. 36 - máx. 100 anos). A média de idade do sexo feminino foi de 69,8 anos, e a do sexo masculino de 66,5 anos. O tempo de internação médio foi de 5,5 dias, tendo sido a internação mais curta de dois dias e a mais longa de 19 dias. Quatro dos 51 pacientes evoluíram a óbito durante a internação. A comorbidades mais frequentemente encontradas foram a hipertensão arterial sistêmica (HAS), seguido de tabagismo e fibrilação atrial (Tabela 1).
Tabela 1. Características dos pacientes e dados laboratoriais

\begin{tabular}{lc}
\hline & $\begin{array}{c}\text { Média (\%) } \\
\text { ou } \pm \text { DP }\end{array}$ \\
\hline Características gerais & $68 \pm 15$ \\
Idade & $24(47,1)$ \\
Sexo: Feminino & $27(52,9)$ \\
$\quad$ Masculino & \\
Comorbidades & $40(78,4)$ \\
HAS & $34(66,7)$ \\
Tabagismo & $18(35,3)$ \\
Fibrilação atrial & $15(29,4)$ \\
Insuficiência cardíaca & $14(27,5)$ \\
Diabete melito & $11(21,6)$ \\
Doença arterial obstrutiva periférica & $8(15,7)$ \\
Dislipidemia & $7(13,7)$ \\
Doença coronariana & $6(11,8)$ \\
Doença renal crônica & $6(11,8)$ \\
Etilismo & $5(9,8)$ \\
Infarto agudo do miocárdio prévio & $3(5,9)$ \\
Cardiopatia & \\
\hline
\end{tabular}

Legenda: DP, desvio-padrão; HAS, hipertensão arterial sistêmica.

Com relação ao tempo de evolução da doença, ou seja, o tempo desde o início dos sintomas até o atendimento hospitalar, foi constatado que: $17,64 \%$ dos pacientes foram atendidos num período menor que 12 horas do início dos sintomas; $11,76 \%$ foram atendidos nas primeiras $24 \mathrm{~h}$ dos sintomas; $21,56 \%$ procuraram o serviço entre 24 e $72 \mathrm{~h}$ dos sintomas; $43,13 \%$ dos pacientes apresentaram evolução de $72 \mathrm{~h}$ a 4 semanas até a procura do serviço; e, por fim, 5,88\% dos pacientes apresentaram uma progressão da doença em um período maior do que 4 semanas.

Os pacientes foram divididos em dois grupos, os submetidos ou não à amputação de membro. Das 16 amputações realizadas, $62,5 \%$ foram primárias, ou seja, não precedidas por tentativa de revascularização do membro. Das amputações realizadas que sucederam uma abordagem prévia (correspondendo a 37,5\%), em 50\% dos casos foi realizado angioplastia percutânea, em 33,3\% dos casos foi realizado embolectomia, e em $16,6 \%$ dos casos foi realizado trombectomia associada a endarterectomia.

Não houve diferença estatisticamente significativa nos exames laboratoriais colhidos na internação, quando comparados o grupo que evoluiu para amputação e o grupo que teve o membro preservado. A leucocitose, porém, foi encontrada com valores mais elevados no grupo de pacientes com desfecho de amputação $(\mathrm{p}=0,0568)$ (Tabela 2). 
Moraes-Silva MA, et al. Análise epidemiológica das oclusões arteriais agudas dos membros inferiores.

Tabela 2. Exames laboratoriais distribuídos conforme desfecho clínico

\begin{tabular}{lccc}
\hline & \multicolumn{2}{c}{ Média \pm DP ou valor absoluto } & $\mathrm{p}^{*}$ \\
\cline { 2 - 3 } & Amputados & Não amputados & 0,6556 \\
\hline Hemoglobina $(\mathrm{g} / \mathrm{dL})$ & $12,65 \pm 2,76$ & $13,02 \pm 2,08$ & 0,0568 \\
Leucócitos & $13.212 \pm 3.443$ & $10.433 \pm 4.149$ & 0,1937 \\
Creatinina sérica & $1,76 \pm 1,65$ & $1,26 \pm 0,63$ & 0,2719 \\
TAP & $78 \% \pm 25 \%$ & $69 \% \pm 18 \%$ & 0,200 \\
\hline
\end{tabular}

Legenda: DP, desvio-padrão; TAP, tempo de atividade de protrombina. * Teste t de Student.

A distribuição dos pacientes segundo o quadro clínico de apresentação está ilustrada na Figura 1. Podemos observar que a categoria II b (ameaça imediata do membro) foi a mais prevalente, correspondendo a dois terços dos casos.

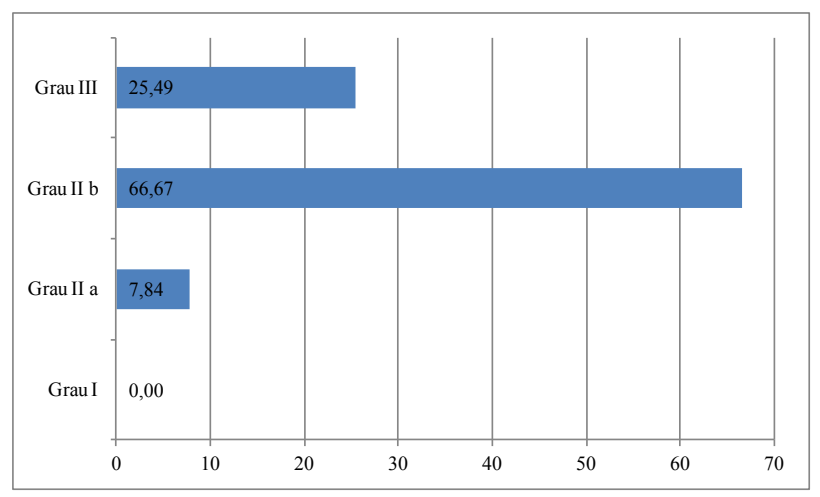

Figura 1. Distribuição do quadro clínico dos pacientes segundo a classificação de Rutherford. Nenhum paciente era assintomático (Grau I)

A distribuição do nível da oclusão arterial, baseado nas características clínicas e palpação dos pulsos, está ilustrado na Figura 2, sendo mais da metade (51\%) correspondendo a obstruções femorodistais.

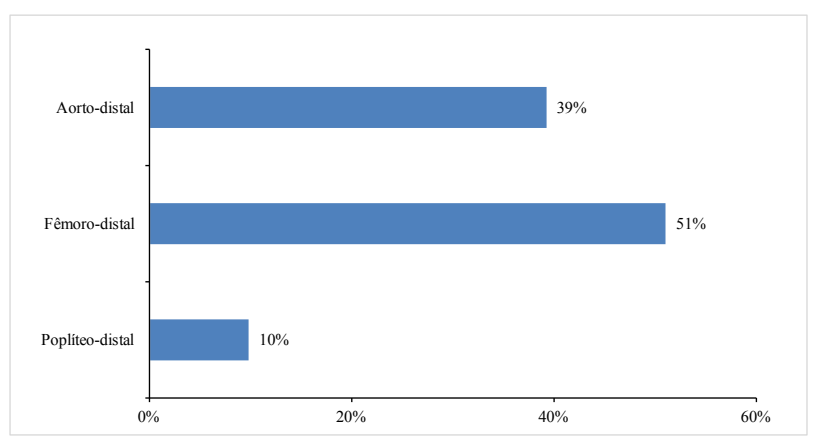

Figura 2. Classificação das oclusões arteriais pela altura da oclusão
Com relação ao tipo de procedimento cirúrgico realizado, o mais frequente foi a tromboembolectomia, seguido pela amputação transfemoral (Figura 3). No total, foram realizadas 25 tromboembolectomias, correspondendo a $39,7 \%$ dos procedimentos. Todas as amputações corresponderam a $25,4 \%$ das cirurgias tendo sido 12 amputações transfemorais (19,1\%), 2 amputações transtibiais $(3,2 \%)$ e 2 amputações transfalangeanas $(3,2 \%)$. O tratamento endovascular apresentou pouca indicação $(12,7 \%)$, recaindo na cirurgia aberta a opção de revascularização imediata do membro mais utilizada.

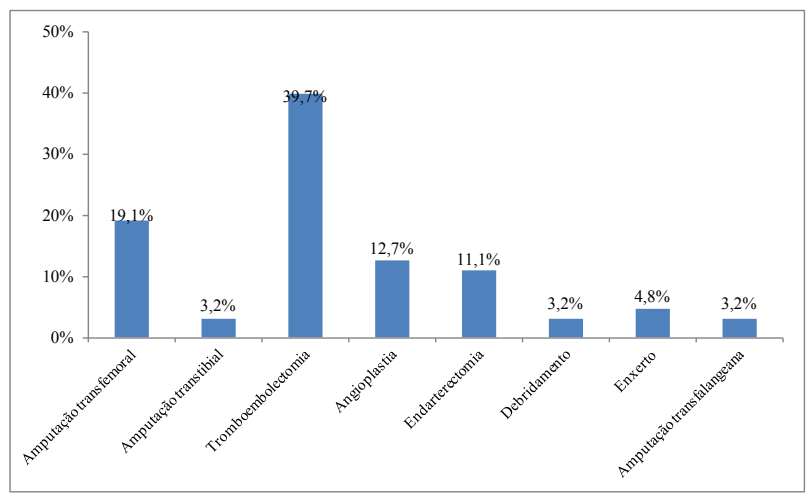

Figura 3. Porcentagem dos procedimentos cirúrgicos efetuados

A Tabela 3 descreve a análise comparativa entre os fatores de risco entre os pacientes submetidos ou não à amputação. Não houve diferença estatística entre os grupos. Entretanto, a doença arterial obstrutiva periférica foi mais prevalente no grupo de pacientes submetidos à amputação ( 40 vs 13,9 $\%$ ), apresentando valores de $\mathrm{p}$ mais próximos da significância estatística $(\mathrm{p}=0,1484)$.

Não houve diferença estatística significativa nas comorbidades entre os grupos que evoluíram ou não para amputação. No entanto, os pacientes classificados como Rutherford III apresentaram índices maiores de amputação $(\mathrm{p}=0.0043)$. 
Tabela 3. Comparação entre os fatores de risco entre os grupos de pacientes amputados e não amputados

\begin{tabular}{lccc}
\hline $\mathbf{t}$ & Amputados & Não amputados & $\mathbf{p}$ \\
\hline Hipertensão & $80 \%$ & $77,8 \%$ & 1.0000 \\
Tabagismo & $66,7 \%$ & $66,7 \%$ & 0.7379 \\
DAOP & $40 \%$ & $13,9 \%$ & 0.1484 \\
FA & $33,3 \%$ & $36,1 \%$ & 1.0000 \\
Diabete melito & $26,7 \%$ & $27,8 \%$ & 0.7338 \\
IC & $20 \%$ & $33,3 \%$ & 0.7302 \\
IAM prévio & $13,3 \%$ & $8,3 \%$ & 0.5915 \\
Etilismo & $13,3 \%$ & $11,1 \%$ & 0.6377 \\
Dislipidemia & $13,3 \%$ & $16,7 \%$ & 1.0000 \\
Doença renal crônica & $13,3 \%$ & $11,1 \%$ & 0.6377 \\
Doença coronariana & $6,7 \%$ & $16,7 \%$ & 0.6618 \\
Hipertensão + tabagismo & $53,8 \%$ & $52,6 \%$ & 1.0000 \\
Hipertensão + diabete melito & $30,8 \%$ & $23,7 \%$ & 0.7161 \\
\hline
\end{tabular}

IC: insuficiência cardíaca; IAM: infarto agudo do miocárdio; DAOP: doença arterial obstrutiva periférica; FA: fibrilação atrial.

\section{DISCUSSÃO}

Trabalhos recentes sobre a epidemiologia das doenças arteriais periféricas apontam o tabagismo como o fator de risco que mais contribui sua ocorrência. Além disso, é apontado que o tabagismo parece ter um papel mais proeminente para o desenvolvimento da doença arterial periférica do que outras doenças ateroscleróticas. Em um estudo de coorte conduzido por Fowkes et al. ${ }^{16}$, o cigarro foi associado a um risco relativo altamente significativo para doenças arteriais comparando-se com outras doenças cardiovasculares. O tabagismo foi o único fator de risco tradicional para o qual houve diferença significativa da odds ratio entre doença arterial periférica e outras doenças cardiovasculares ${ }^{10}$.

No presente estudo, o tabagismo aparece como o segundo fator de risco mais prevalente nas oclusões arteriais agudas, precedido pela hipertensão. No entanto, apesar da alta prevalência de fatores de risco, como o diabete e hipertensão nos pacientes amputados, não se observou correlação entre a presença desses fatores com a perda do membro.

Ainda de acordo com Criqui e Aboyans ${ }^{10}$, a evolução da doença arterial periférica em diabéticos se mostrou ser mais desfavorável. Em um estudo realizado por Jude et al. ${ }^{17}$, os pacientes diabéticos com doença arterial periférica tinham cinco vezes mais probabilidade de sofrer uma amputação do que outros pacientes não diabéticos, além de três vezes mais chance de mortalidade. Há evidência também de uma distribuição anatômica diferente da doença, com maior acometimento de artérias distais em comparação aos não diabéticos. Além da isquemia, a infecção tem um papel importante, agravando o risco de amputação nesses pacientes ${ }^{8}$. Outros estudos identificaram os fatores de risco mais importantes para isquemia crítica em pacientes com doença arterial crônica: tabagismo, diabete melito e doença renal terminal exigindo diálise ${ }^{18}$.

Na presente casuística, uma pequena porcentagem dos pacientes eram diabéticos $(27,5 \%)$, não estando este fator de risco relacionado à perda do membro. A aterosclerose difusa no diabete parece então estar mais relacionada à evolução da doença obstrutiva crônica do que os casos de embolia e/ou trombose arterial aguda. A doença renal crônica também não foi prevalente no presente estudo, correspondendo somente a $11,8 \%$ dos casos. A presença de fibrilação atrial foi pequena e, por consequência, a prevalência de OAA de origem cardioembólica. Isso leva a supor que os casos de embolia cardiogênica foram suplantados pelas tromboses arteriais em pacientes ateroscleróticos crônicos. Isso é reforçado pela maior ocorrência de oclusões infra-inguinais, setor mais comumente acometido pela doença arterial crônica.

Em estudo comparativo entre pacientes submetidos a revascularização cirúrgica ou endovascular, os principais fatores preditores para a perda do membro foram a doença coronariana e apresentação clínica grau III da Classificação de Rutherford ${ }^{9}$. Apesar da coronariopatia não ser prevalente no presente estudo, observamos que a dor isquêmica de repouso foi a característica clínica mais encontrada.

A cirurgia aberta ainda foi a mais empregada no tratamento da OAA. Mesmo com o advento das técnicas endovasculares e fácil acesso à equipe especializada, a 
tromboembolectomia parece ser o tratamento com mais possibilidade de salvamento de membro pelo cirurgião assistente ${ }^{19}$.

Houve algumas limitações durante este estudo. A primeira delas é que se trata de um estudo retrospectivo em sua natureza. Uma limitação adicional é não padronização de dados registrados em prontuários, o que restringiu algumas possibilidades de análises por ausência de variáveis.

\section{CONCLUSÃO}

O sintoma inicial dos pacientes com oclusão arterial aguda foi a dor isquêmica de repouso, com melhora clínica significativa com o tratamento cirúrgico clássico na maior parte dos casos. Existe elevada prevalência de fatores de risco para doença arterial periférica em pacientes com oclusão arterial aguda, principalmente hipertensão e tabagismo, porém, estes não foram diretamente relacionadas com a evolução para amputação do membro.

Indicação sobre as contribuições de autoria: Moraes-Silva MA: Concepção e desenho do estudo, Análise e interpretação dos dados, Redação do manuscrito, Revisão crítica do texto, aprovação final do manuscrito, Análise estatística, Responsabilidade geral pelo estudo; Bueno G: Análise e interpretação dos dados, Coleta de dados, Redação do manuscrito, Revisão crítica do texto; Jesus-Silva SG: Revisão crítica do texto; Aprovação final do manuscrito; Análise estatística; Krupa AE, Cardoso RS: Revisão crítica do texto; Aprovação final do manuscrito.

\section{REFERÊNCIAS}

1. Mansur AP, Favarato D. Mortalidade por doenças cardiovasculares no Brasil e na região metropolitana de São Paulo: atualização 2011. Arq Bras Cardiol. 2012;99(2):75561. doi: 10.1590/S0066-782X2012005000061.

2. Korabathina R, Weintraub AR, Price LL, Kapur NK, Kimmelstiel CD, Iafrati MD, Ali Tahir SM. Twentyyear analysis of trends in the incidence and in-hospital mortality for lower-extremity arterial thromboembolism. Circulation. 2013;128(2):115-21. doi: 10.1161/ CIRCULATIONAHA.113.003543.

3. Acute limb ischemia: definition and nomenclature for acute limb ischemia. etiology, clinical presentation and clinical evaluation. J Vasc Surg. 31(1):S135-40. doi: 10.1016/S07415214(00)81018-6

4. Duval S, Keo HH, Oldenburg NC, Baumgartner I, Jaff MR. The impact of prolonged lower limb ischemia on amputation, mortality, and functional status : The FRIENDS registry. Am Heart J. 2010;168(4):577-87. doi: 10.1016/j.ahj.2014.06.013.

5. Gabriel S, Serafim P. Doença arterial obstrutiva periférica e índice tornozelo-braço em pacientes submetidos à angiografia coronariana. Braz J Cardiovasc Surg. 2007;22(1):49-59. doi: 10.1590/S0102-76382007000100011.

6. Vaz C, Duarte V, Santos A. Doença arterial periférica e qualidade de vida. Angiol Cir Vasc. 2013;9:1-7. Disponível em: http://www.scielo.mec.pt/scielo.php? script=sci_ arttext\&pid=S1646-706X2013000100003.

7. Varu VN, Hogg ME, Kibbe MR. Critical limb ischemia. JVasc Surg. 2010;51(1):230-41. doi: 10.1016/j.jvs.2009.08.073.

8. Wassel CL, Loomba R, Ix JH, Allison MA, Denenberg JO, Criqui MH. Family history of peripheral artery disease is associated with prevalence and severity of peripheral artery disease: the San Diego population study. J Am Coll Cardiol. 2011;58(13):1386-92. doi:10.1016/j.jacc.2011.06.023
9. Rutherford RB, Baker JD, Ernst C, Johnston KW, Porter JM, Ahn S, Jones DN. Recommended standards for reports dealing with lower extremity ischemia: revised version. J Vasc Surg. 1997;26:517-38. doi: 10.1016/S0741-5214(97)70045-4.

10. Criqui MH, Aboyans V. Epidemiology of peripheral artery disease. Circ Res. 2016;116:1509-26. doi: 10.1161/ CIRCRESAHA.116.303849.

11. Lawrence PF, Chandra A. When should open surgery be the initial option for critical limb ischaemia? Eur J Vasc. 2010;39(Suppl. 1):S32-S37. doi: 10.1016/j.ejvs.2009.11.032.

12. Malta M, Cardoso LO, Bastos FI, Magnanini MMF, Silva CMFP. Iniciativa STROBE: subsídios para a comunicação de estudos observacionais. Rev Saude Publica. 2010;44(3):55965. http://dx.doi.org/10.1590/S0034-89102010000300021.

13. Menezes J, Fernandes e Fernandes J, Santos Carvalho C, Barbosa J, Mansilha A. Estudo da prevalência da doença arterial periférica em Portugal. Angiol Cir Vasc. 2009;5:59-68. Disponível em: http://spacv.org/wp-content/ uploads/2015/06/02_v5-02_artigo-original.pdf.

14. Arango HG. Bioestatística teórica e computacional. 2a ed. Rio de Janeiro: Guanabara Koogan; 2005.

15. Pavão ALB, Andrade D, Mendes W, Martins M, Travassos C. Estudo de incidência de eventos adversos hospitalares, Rio de Janeiro, Brasil: avaliação da qualidade do prontuário do paciente. Rev Bras Epidemiol. 2010;14(4):651-61. doi: 10.1590/S1415-790X2011000400012.

16. Fowkes FG, Rudan D, Rudan I, Aboyans V, Denenberg JO, McDermott MM, Norman PE, Sampson UK, Williams LJ, Mensah GA, Criqui MH. Comparison of global estimates of prevalence and risk factors for periph- eral artery disease in 2000 and 2010: a systematic review and analysis. Lancet. 2013;382:1329-40. doi: 10.1016/S0140-6736(13)61249-0.

17. Jude EB, Oyibo SO, Chalmers N, Boulton AJ. Peripheral 
arterial disease in diabetic and nondiabetic patients: a comparison of severity and out- come. Diabetes Care. 2001;24:1433-7. doi.org/10.2337/diacare.24.8.1433.

18. Shirasu T, Miyata T, Nishiyama A, Miyahara T, Hoshina K, Shigematsu K, Watanabe T. Useful predictors for critical limb ischemia in severely ischemic limbs. Int Angiol.
2016;35(5):460-8.

19. Taha AG, Byrne RM, Avgerinos ED, Marone LK, Makaroun MS, Chaer RA. Comparative effectiveness of endovascular versus surgical revascularization for acute lower extremity ischemia. J Vasc Surg. 2015;61(1):147-54. doi: 10.1016/j. jvs.2014.06.109.

Artigo recebido em: 24.03.17

Artigo aceito em: 17.10.17 